\title{
Investigation of Skin Barrier Functions and Allergic Sensitization in Patients with Hyper-IgE Syndrome
}

\author{
Gábor Mócsai $^{1,2}$ - Krisztián Gáspár ${ }^{1,2} \cdot$ Zsolt Dajnoki $^{1,2} \cdot$ Beáta Tóth $^{3} \cdot$ Edit Gyimesi $^{4}$. \\ Tamás Bíró $^{5}$ • László Maródi ${ }^{3}$ - Andrea Szegedi ${ }^{1,2}$
}

Received: 12 June 2015 / Accepted: 17 September 2015 /Published online: 9 October 2015

(C) Springer Science+Business Media New York 2015

\begin{abstract}
Purpose Hyper-IgE syndrome (HIES) is a severe primary immunodeficiency, characterized by increased serum IgE levels as well as recurrent infections and atopic dermatitis (AD)-like skin lesions. $\mathrm{AD}$ is a chronic inflammatory skin disease with immunologic alterations (Th2-Th22 polarization) and characteristic skin barrier dysfunctions. Our aim was to investigate physicochemical skin barrier alterations and allergic sensitization in STAT3-HIES patients in order to explore whether skin barrier dysfunction can play a role in the eczematoid skin lesions in these patients.

Methods In our experiments STAT3 and FLG mutation analyses were performed in STAT3-HIES $(n=7)$ and AD $(n=49)$ patients. Laboratory parameters (LDH and Eos counts), immunologic alterations (Th17 cell counts), allergic sensitization (total and specific IgE levels, skin prick tests, and medical history records), skin barrier changes [transepidermal water loss (TEWL), skin $\mathrm{pH}]$, serum and stratum corneum thymic stromal lymphopoietin (TSLP) levels were also examined.
\end{abstract}

Andrea Szegedi

aszegedi@med.unideb.hu

1 Division of Dermatological Allergology, Faculty of Medicine, University of Debrecen, Nagyerdei krt. 98., Debrecen 4032, Hungary

2 Department of Dermatology, Faculty of Medicine, University of Debrecen, Debrecen, Hungary

3 Department of Infectious and Pediatric Immunology, Faculty of Medicine, University of Debrecen, Debrecen, Hungary

4 Institute of Laboratory Medicine, Faculty of Medicine, University of Debrecen, Debrecen, Hungary

5 DE-MTA “Lendület” Cellular Physiology Research Group, Department of Physiology, Faculty of Medicine, University of Debrecen, Debrecen, Hungary
Results Impaired Th17 cell numbers, but normal physicochemical barrier functions, as well as serum and stratum corneum TSLP levels, were found in STAT3-HIES, while these parameters were significantly altered in AD patients. Allergic sensitization was detected in nearly all AD patients, while no signs of sensitization occurred in STAT3-HIES.

Conclusions Our study demonstrated that the skin barrier functions of STAT3-HIES patients are not damaged and they differ significantly from the altered skin barrier functions of AD patients. A well-functioning physicochemical skin barrier may be one of the explanations on the contradiction between the extremely high total IgE levels and the lack of allergic sensitization in these patients. Our study underlines the importance of skin barrier in the development of allergic sensitization.

Keywords Atopic dermatitis · filaggrin · hyper- $\operatorname{IgE}$ syndrome $\cdot$ skin barrier

\section{Introduction}

Hyper IgE syndrome (HIES) is a rare, complex, primary immunodeficiency disorder with susceptibility to infections caused by extracellular bacteria and fungi and also occurs with atopic manifestations, such as extremely high serum $\operatorname{IgE}$ levels and eczema [1]. It has been established that HIES is a polysystemic disease in which not only the immune system is aberrant, but skeletal and connective tissue abnormalities are characteristic features [2]. Several investigations have revealed that most patients with HIES have a dominant negative mutation in the signal transducer and activator of transcription 3 (STAT3) gene, while in a minority of cases homozygous null mutations in the dedicator of cytokinesis 8 (DOCK8), in phosphoglucomutase 3 (PGM3) genes cause autosomal recessive 
HIES associated with mycobacterial and viral infections [3-7]. Although the underlying genetic defects are mostly described in HIES, the knowledge of the molecular pathogenesis in the background of the clinical symptoms, including the development of eczematoid skin lesions, which are often diagnosed as atopic dermatitis (AD)-like skin lesions, is limited [8-10].

$\mathrm{AD}$ is a common chronic inflammatory skin disease that is frequently accompanied by other atopic diseases such as allergic rhinitis and bronchial asthma and is also associated with elevated serum IgE levels [11]. Based on previous results, immunological alterations and skin barrier dysfunctions are indicated as important factors in the complex etiological background of AD [12]. Physicochemical skin barrier defects can be the results of genetic mutations, such as the wellcharacterized filaggrin (FLG) haploinsufficiency or copy number variations, or can also be an acquired manifestation that occurs after the harmful influence of several environmental agents [13]. This barrier impairment is a critical and early factor in the development of inflammatory skin lesions in $\mathrm{AD}$ and also a crucial event in allergic sensitization, which is a characteristic feature of this disease [14].

In the present study our aim was to investigate for the first time the physicochemical skin barrier alterations and allergic sensitizations in STAT3-HIES patients in order to compare them with those of AD patients and to find similar or different pathogenetic events in the development of eczematoid skin lesions in these two disorders. Importantly, we also intended to strengthen the indirect connection between the physicochemical skin barrier and allergic sensitization.

\section{Materials and Methods}

\section{Patients}

Peripheral blood was obtained from 7 Caucasian patients with STAT3-HIES, 3 males and 4 females (mean age: 16 years, range: 4-40 years) and from 49 patients suffering from $\mathrm{AD}$ with mild-to-moderate clinical symptoms. Patients with STAT3-HIES and AD did not suffer from any concomitant skin diseases at the time of the examination and had not been treated with any moisturizers for 1 day, topical corticosteroids or calcineurin inhibitors for 3 days, and with systemic immunosuppressants for 28 days prior to examination. The following biochemical parameters were examined on these two populations: eosinophil count, serum lactate dehydrogenase (LDH) level, total IgE and specific IgE levels (against house dust mites, ragweed and cat dander antigens). Data on the patients' history of other allergic diseases and sensitization were recorded. The severity of $\mathrm{AD}$ was determined using OSCORAD (Objective SCORing Atopic Dermatitis). The local skin inflammation of STAT3-HIES and AD skin lesions was assessed by the TIS (Three Item Severity) scoring system. Healthy controls $(n=10)$ were included as the basis for the comparison of barrier functions. All participants provided written informed consent according to the principles of the Declaration of Helsinki. The study was approved by the local ethics committee.

\section{Filaggrin and STAT3 Genotyping}

Analysis of the most common FLG mutations R501X and 2282del4 was performed for all STAT3-HIES and AD patients, and STAT3 for STAT3-HIES patients. DNA isolated from peripheral blood mononuclear cells with GenElute Blood Genomic DNA Kit (Sigma, Chemical Co., St. Louis, $\mathrm{MO}$, USA) was subjected to polymerase chain reaction (PCR) amplification. All PCR products were purified with QIAquick PCR purification Kit (Qiagen, Inc) and bidirectionally sequenced on an ABI Prism 3100 automated sequencer with Big-Dye terminator cycle sequencing reagents (Applied Biosystems, Foster City, CA).

\section{Evaluation of Intracellular IL-17 by Flow Cytometry}

Measurement of IL-17 producing T cells was performed to strengthen the diagnosis of STAT3-HIES patients as described previously [15].

\section{Measurement of Transepidermal Water Loss (TEWL) and Skin pH}

Measurements were performed under standardised laboratory conditions at a temperature of $22-25^{\circ} \mathrm{C}$ and a humidity level of $40-60 \%$. Before the measurements, patients were allowed to adapt to the room conditions for $5 \mathrm{~min}$. TEWL measurements $\left(\mathrm{g} / \mathrm{hm}^{2}\right)$ were carried out with Tewameter TM300 (Courage and Khazaka, Cologne, Germany) on nonlesional (flexural forearm) and on lesional skin of patients. The duration of the measurements, performed in triplicates, was $30 \mathrm{~s}$. Skin pH measurements were carried out with pH 905 (Courage and Khazaka, Cologne, Germany) on nonlesional (flexural and extensor forearm), and lesional skin of patients. The measurements were repeated 20 times, and the last value was recorded, which was shown to be the steady state.

\section{TSLP ELISA}

Serum was isolated from patients and aliquoted, and the TSLP levels were determined using the ELISA Human TSLP Quantikine Immunoassay according to the manufacturer's instructions (R\&D Systems, Minneapolis, MN). 


\section{Measurement of the Stratum Corneum TSLP Concentration}

The tape-stripping method and measurement of the stratum corneum TSLP expression level were carried out according to the method described in a previous report by Sano et al. [16].

\section{Skin Prick Test}

Skin prick test was performed with commercial test solutions of ragweed, cat dander, house dust mite, peanut, milk, egg white, and wheat (all from Lofarma S.p.A., Milan, Italy). Reaction intensity was reported as described by manufacturer's instruction.

\section{Statistical Analysis}

To determine the statistical significance of the results, the Kruskal-Wallis test and the Mann-Whitney test were used to analyse nonparametric distributions, and Fisher's exact test was applied to compare specific IgE values and the history of sensitization. $P$-values $<0.05$ were considered statistically significant $(* p<0.05 ; * * p<0.01 ; * * * p<0.005)$.

\section{Results}

\section{Clinical and Immunological Characteristics of STAT3-HIES Patients}

In this study, we diagnosed 7 patients with STAT3-HIES on the basis of National Institutes of Health-HIES (NIH-HIES) score (Table 1), STAT3 mutation analysis, IL-17 production of $\mathrm{T}$ helper cells, and total IgE levels. All 7 patients showed characteristic clinical and laboratory manifestation of STAT3-HIES with the range of NIH scores between 44 and 70 points. The H332Y STAT3 mutation was detected in 3 patients, and the R382W STAT3 mutation was identified in 4 patients. The mean number of IL-17-producing Th17 was significantly decreased in STAT3-HIES patients $(0.12 \%$ of $\mathrm{CD}^{+} \mathrm{T}$ cells $)$ compared with healthy controls $(0.99 \%$ of $\mathrm{CD}^{+} \mathrm{T}$ cells). Total IgE levels were significantly higher in patients with STAT3-HIES (mean: $8441 \mathrm{kU} / \mathrm{L}$ ) compared to healthy controls. In our AD patients, normal IL-17-producing Th17 cell counts were measured ( $1.006 \%$ of $\mathrm{CD} 4^{+} \mathrm{T}$ cells), but significant increases in mean total IgE levels were found (mean: $3858 \mathrm{kU} / \mathrm{L}$ ) (Table 2).

\section{Skin Barrier Functions in STAT3-HIES and AD Patients}

The TIS scoring system showed that the local severity of skin lesions in STAT3-HIES and AD patients are comparable
(Table 2). None of the STAT3-HIES patients carried the two most common FLG mutations (R501X and 2282del4), which were present in $30.5 \%$ of patients with AD. TEWL and skin $\mathrm{pH}$ were measured on nonlesional skin of healthy controls as well as of patients with STAT3-HIES and AD, and on lesional skin of all patients. TEWL on nonlesional skin showed no difference between the STAT3-HIES patients and healthy controls, but the AD patients had significantly higher values (Fig. 1a). On lesional skin, significant elevation in TEWL was detected in the AD patients compared to the STAT3HIES group (Fig. 1b). Measuring $\mathrm{pH}$ on nonlesional skin, the flexural forearm of STAT3-HIES patients showed significant increase compared to healthy controls, but no significance was detected on the extensor part. The $\mathrm{pH}$ of nonlesional skin in the AD patient group showed significant increases both on flexural and extensor parts compared to healthy controls and STAT3-HIES patients (Fig. 1c-d). On lesional skin, significant increase in $\mathrm{pH}$ were detected in $\mathrm{AD}$ patients compared to STAT3-HIES patients (Fig. 1e).

\section{Serum and Stratum Corneum TSLP Content}

When determining serum and stratum corneum TSLP levels in the three groups, no significant differences in serum and stratum corneum TSLP levels were detected between the STAT3-HIES patients and the healthy controls, but significant increases were found in $\mathrm{AD}$ patients compared to the other two groups (Fig. 2a-b).

\section{Allergic Sensitization}

Similarly to the barrier measurements, our data indicated that the level of allergic sensitization differed profoundly between AD and STAT3-HIES patients. AD patients showed significantly elevated specific IgE levels against aeroallergens (cat dander, ragweed and house dust mite) (Fig. 3a) compared to the STAT3-HIES patient group, while no relevant specific IgE level could be detected in the latter group. Skin prick test of the STAT3-HIES patients displayed no positivity (data not shown). In the personal medical history of the patients, the occurrence of allergic asthma and rhinitis was observed significantly higher in the AD group than in the STAT3-HIES group (Fig. 3b).

\section{Discussion}

Cutaneous manifestations of STAT3-HIES are newborn rash, Staphylococcus aureus-caused cold abscesses, mucocutaneous candidiasis and eczematoid lesions, which are often referred to as AD-like eruptions [17-19]. The eczema observed in STAT3-HIES is similar to AD clinically (papular, pruritic lesions with lichenification) and histopathologically, and both 
Table 1 NIH scoring system of STAT3-HIES patients

\begin{tabular}{|c|c|c|c|c|c|c|c|}
\hline & Patient 1 & Patient 2 & Patient 3 & Patient 4 & Patient 5 & Patient 6 & Patient 7 \\
\hline Highest IgE (IU/ml) & 10 & 10 & 10 & 10 & 10 & 10 & 10 \\
\hline Skin abscesses & 8 & 8 & 2 & 4 & 4 & 4 & 0 \\
\hline Pneumonia & 8 & 6 & 4 & 2 & 2 & 8 & 8 \\
\hline Lung parenchymal abnormality & 0 & 0 & 0 & 0 & 0 & 0 & 6 \\
\hline Other serious infection & 4 & 4 & 0 & 0 & 0 & 0 & 4 \\
\hline Fatal infection & 0 & 0 & 0 & 0 & 0 & 0 & 0 \\
\hline Highest eosinophylia & 0 & 0 & 0 & 0 & 3 & 0 & 0 \\
\hline Neborn rash & 4 & 4 & 4 & 0 & 0 & 4 & 4 \\
\hline Eczema & 4 & 2 & 2 & 4 & 4 & 2 & 0 \\
\hline Sinusitis/otitis (times/worst year) & 4 & 4 & 2 & 0 & 0 & 1 & 4 \\
\hline Candidiasis & 2 & 0 & 2 & 1 & 1 & 1 & 1 \\
\hline Retained primary teeth & 2 & 0 & 2 & 2 & 2 & 2 & 0 \\
\hline Scoliosis/max curve & 0 & 0 & 0 & 2 & 2 & 2 & 0 \\
\hline Pathologic fracture & 8 & 0 & 0 & 0 & 0 & 0 & 0 \\
\hline Hyperextensibility & 4 & 4 & 4 & 4 & 4 & 4 & 4 \\
\hline Characteristic face & 5 & 5 & 5 & 5 & 5 & 5 & 5 \\
\hline Interalar distance & 0 & 0 & 0 & 0 & 0 & 0 & 0 \\
\hline High palate & 0 & 2 & 2 & 2 & 2 & 2 & 2 \\
\hline Midline anomaly & 0 & 5 & 0 & 5 & 5 & 5 & 0 \\
\hline Lymphoma & 4 & 0 & 0 & 0 & 0 & 0 & 0 \\
\hline Young age add-on at onset (AD) & 7 & 7 & 7 & 5 & 5 & 5 & 7 \\
\hline Total & 74 & 61 & 46 & 46 & 49 & 55 & 55 \\
\hline
\end{tabular}

diseases are associated with eosinophilia and high serum $\operatorname{IgE}$ levels, but there are also several distinctions. The skin involvement in STAT3-HIES is mostly confined to the face and extensor surfaces of the extremities, while in $\mathrm{AD}$, flexural surfaces are typically involved. Staphylococcal infections are common in both diseases, but in AD they are usually superficial, whereas in STAT3-HIES cold abscesses may develop
[20]. Mucocutaneous candidiasis is a characteristic feature of STAT3-HIES, but not that of AD. While high serum level of allergen specific IgE is nearly absent in STAT3-HIES, AD is often associated with severe sensitization and accompanied by food allergy, asthma or allergic rhinitis [12]. In HIES the role of Th17 cells is well-known, however, its contribution to the development of eczematoid skin lesions remains unclear [21].
Table 2 Clinical parameters, laboratory data (routine and immunological), and mutations of healthy controls (HC), STAT3-HIES and AD patients

\begin{tabular}{llll}
\hline & HC & STAT3-HIES & AD \\
\hline Mean age with range (years) & $28(18-46)$ & $16(4-40)$ & $19(4-52)$ \\
Male/female ratio & $5 / 5$ & $3 / 4$ & $22 / 27$ \\
Mean HIES SCORE & N/A & 44.28 & N/A \\
Mean OSCORAD & N/A & 44.28 & N/A \\
Mean TIS SCORE & N/A & 4.71 & 4.85 \\
Mean Eosinophile count (Giga/L) & 0.07 & 0.54 & 0.58 \\
Mean LDH (U/L) & 285.6 & 392.2 & 417.8 \\
Mean Total IgE (kU/L) & 98.4 & 8441 & 3858 \\
Th17 cell numbers (\%) & 0.9933 & 0.1175 & 1.006 \\
H332Y mutation in STAT3 gene & $0 \%(0 / 10)$ & $42 \%(3 / 7)$ & N/A \\
R382W mutation in STAT3 gene & $0 \%(0 / 10)$ & $58 \%(4 / 7)$ & N/A \\
R501X mutation in FLG gene & $0 \%(0 / 10)$ & $0 \%(0 / 7)$ & $8.1 \%(4 / 49)$ \\
2282del4 mutation in FLG gene & $0 \%(0 / 10)$ & $0 \%(0 / 7)$ & $22.4 \%(11 / 49)$
\end{tabular}




\section{A nonlesional skin}

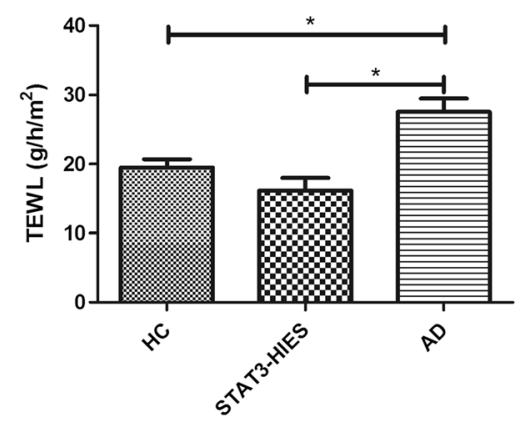

C nonlesional extensor skin

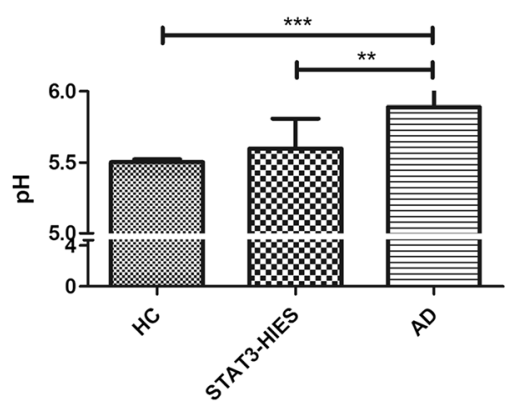

B lesional skin

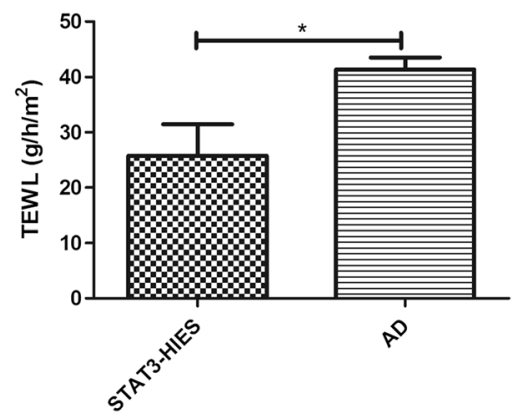

D nonlesional flexural skin E lesional skin

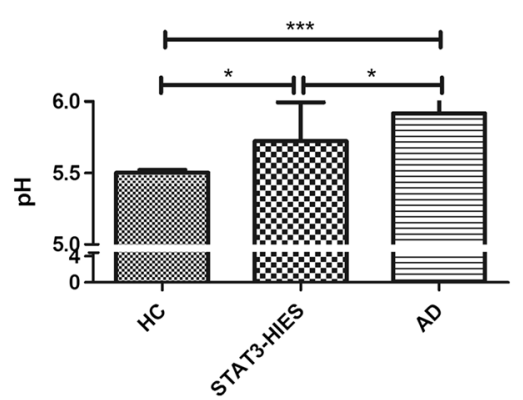

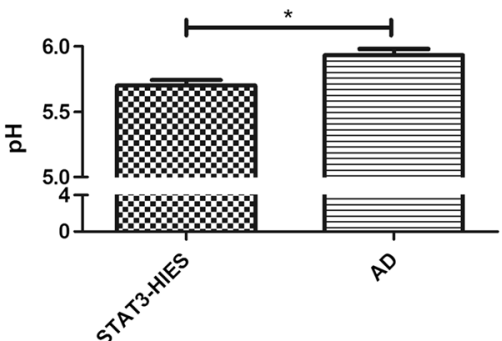

Fig. 1 Skin barrier functions of STAT3-HIES and AD patients. In TEWL no difference was found between the STAT3-HIES patient group and healthy controls on nonlesional skin, but the AD patients had significantly higher values $(P=0.0106$ for STAT3-HIES and $P=0.0152$ for healthy controls) (Fig. 1a). Similarly, on lesional skin significant, elevation in TEWL was observed in the $\mathrm{AD}$ patient group relative to the STAT3-HIES patient group $(P=0.0231)$ (Fig. 1b). Measuring skin $\mathrm{pH}$ on flexural site of nonlesional skin, the STAT3-HIES patient group

Since the eczematoid skin lesions of STAT3-HIES patients are often diagnosed as AD-like skin lesions, and in AD patients cutaneous barrier alterations are considered to be extremely important in the development of skin inflammation and allergic sensitization, our research group intended to

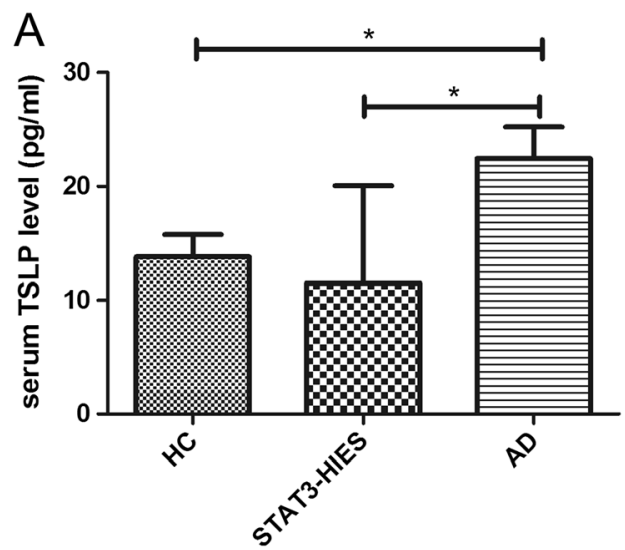

Fig. 2 Serum and stratum corneum (sc) TSLP levels of STAT3-HIES and AD patients. Measuring serum and stratum corneum TSLP levels in the three groups, no significant differences were detected between the STAT3-HIES patients and the healthy controls, but significant increases showed significant increases compared to healthy controls $(P=0.0238)$, but not on extensor site. The $\mathrm{pH}$ of nonlesional skin in $\mathrm{AD}$ patient group showed significant increase compared to healthy controls and STAT3HIES patients $(P=0.0471$ for STAT3-HIES and $P<0.0001$ for healthy controls) (Fig. 1c-d). On lesional skin, significant increases in skin $\mathrm{pH}$ were detected in AD patients compared to STAT3-HIES patients $(P=$ 0.035) (Fig. 1e)

investigate these parameters also in STAT3-HIES patients in order to explore whether physicochemical skin barrier dysfunction has a role in the pathogenesis of skin lesions in HIES.

On the basis of our results, skin barrier seems to function normally in STAT3-HIES patients and does not play a

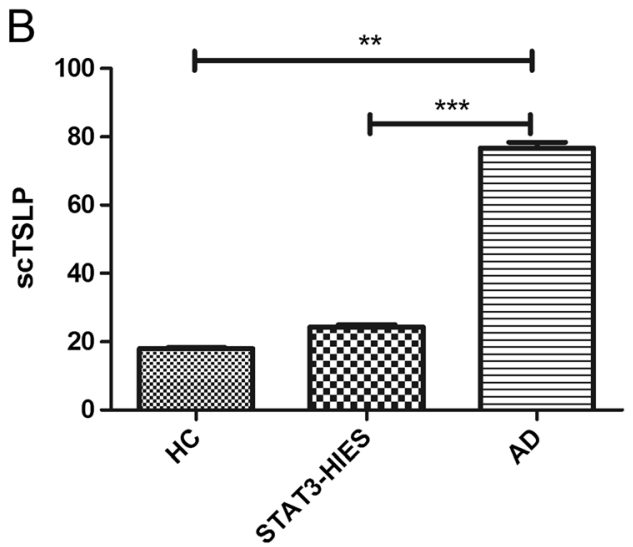

were observed in the AD patient group regarding both parameters when compared to the other two groups (serum TSLP: $P=0.0236$ for healthy controls and $P=0.0329$ for STAT3-HIES; stratum corneum TSLP: $P=$ 0.020 for healthy controls and $P=0.010$ for STAT3-HIES) (Fig. 2a-b) 


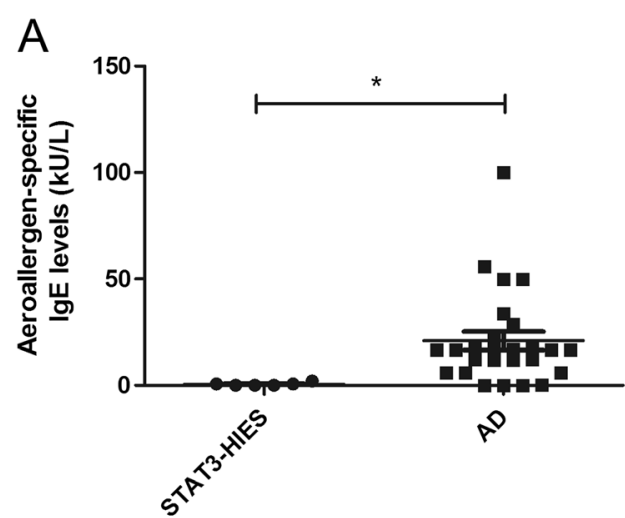

Fig. 3 Allergic sensitization. AD patients showed significantly elevated specific IgE levels against aeroallergens (cat dander, ragweed and house dust mite, $P=0.0339$ (Fig. 3a) compared to STAT3-HIES patient group, where no relevant specific IgE levels could be detected. The occurrence

significant role in the development or maintenance of eczematoid skin lesions. In our study, no differences in TEWL or in skin $\mathrm{pH}$ were observed between healthy controls and STAT3-HIES patients, except in skin $\mathrm{pH}$ on the flexural nonlesional site of the forearm, while significant differences were found between the STAT3-HIES and AD groups for both nonlesional and lesional skin areas. Moreover, no common filaggrin mutations were detected in STAT3-HIES patients, which were present in $34.5 \%$ of our AD patients. Results showing serum and stratum corneum TSLP levels also strengthened our hypothesis, since both were significantly higher in AD group, when compared to healthy controls and STAT3-HIES patients.

In the last few years, measuring TEWL has become one of the most acceptable non-invasive method to examine physicochemical skin barrier alterations [22, 23]. Patients suffering from $\mathrm{AD}$, as well as transgenic mice with barrier gene defects (e.g., CLDN1 and DSG3) show significantly elevated TEWL compared to healthy controls $[24,25]$. In these patients TEWL measured on both lesional and nonlesional skin, showed strong correlations with disease severity; however, during the physicochemical skin barrier recovery this parameter decreased to a normal level. Until now, no TEWL measurements have been performed in STAT3-HIES patients with AD-like skin lesions.

Although skin $\mathrm{pH}$ plays a significant role in normal skin barrier homeostasis, it is influenced not only by the barrier functions, but also via numerous other factors (e.g., sweat and sebum secretion, soaps) $[26,27]$. AD skin shows a more basic $\mathrm{pH}$ than normal skin, caused by the lower amount of FLG breakdown products and the altered skin microbiome [28]. Since it can be affected via the above-mentioned factors, measuring skin $\mathrm{pH}$ is considered to be an indirect method to detect skin barrier alterations. In our study, normal skin $\mathrm{pH}$ was measured in STAT3-HIES patients, except on flexural site of the forearm. This difference may be explained by the altered skin microbiome of STAT3-HIES patients instead of skin barrier disruption, since a recent study published that

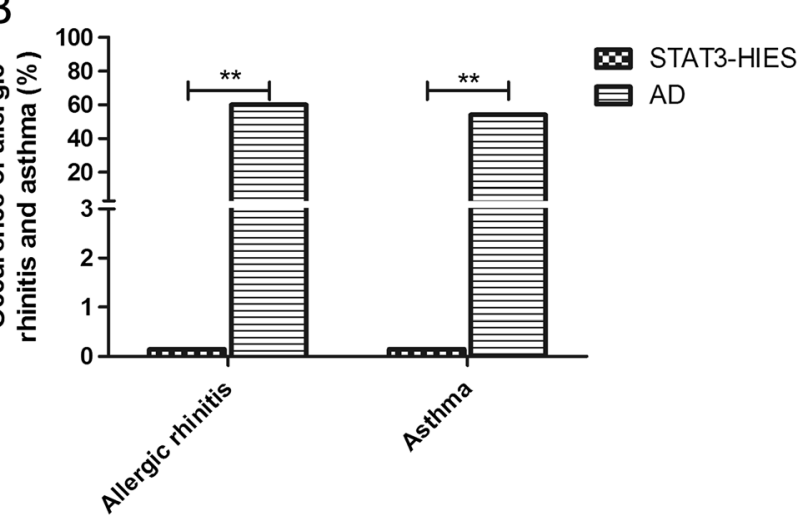

of allergic asthma and rhinitis in the personal medical history was measured significantly less frequently in the STAT3-HIES group than in the $\operatorname{AD}$ group $(P=0.0023$ for rhinitis and $P=0.0030$ for asthma) (Fig. 3b)

the microbiome of these patients contain more Gramnegative bacteria (especially Acinetobacter spp.), which may alter the skin $\mathrm{pH}$ to basic [29].

One of the basic components of the physicochemical barrier is FLG, which has a major role in crosslinking keratin filaments $[13,30]$. Moreover, its degradation products are the natural moisturizing factors (NMFs) that buffer the $\mathrm{pH}$ of the skin and have a role in UV protection (e.g., urocanic acid), and immunomodulation [31]. In European AD patients, basically five (R501X, 2282del4, S3247X, R2447X and 3702delG) FLG null mutations are predisposing to the disease, but in continental regions only R501X and 2282del4 are the most prevalent, which were detected in $34.5 \%$ of our AD patients [30]. Although only 7 STAT3-HIES patients were investigated to these FLG mutations, the lack of these major mutations suggests that FLG haploinsufficiency in the molecular background of eczematoid skin lesions in these patients, probably does not play as important role as it is in $\mathrm{AD}$ patients.

However, the aforementioned methods (TEWL, skin $\mathrm{pH}$, FLG mutations) are well accepted to measure skin barrier functions; the detection of serum and stratum corneum TSLP levels are recently developed methods. Serum TSLP of AD patients and mice with barrier disruption (FATP4 mutation) showed significant increase of these parameters compared to healthy controls, but the direct correlation between serum TSLP levels and disease severity is still unclear [32-34]. A recent investigation postulated that the stratum corneum TSLP level was increased in AD patients, correlated with disease severity (SCORAD), and the authors also suggested that stratum corneum TSLP level could be used as a biomarker of epidermal barrier status [16]. In our study, the serum and stratum corneum TSLP levels were very similar in STAT3-HIES patients compared to healthy controls, which data may also suggest a well-functioning physicochemical skin barrier in the disease and indicate the absence of AD-like skin 
inflammation; on the other hand in AD patients, these TSLP levels were significantly higher compared to HIES patients and healthy controls.

For a long time allergic diseases were considered primarily to be the consequence of alterations in the adaptive immune system (Th1-Th2 imbalance, missing immune deviation or reduced immune suppression), but based on several recent research data dysfunctional physicochemical barriers are now recognized as critical elements in allergic sensitization $[35,36]$. FLG deficiency-mediated barrier disruption represents risk factor for sensitization in $\mathrm{AD}$ and contact dermatitis patients. Other barrier-related gene defects may also predispose to sensitization (SPINK5 - Netherton's syndrome, CDSN - Peeling skin syndrome) [37, 38].

In spite of the high IgE levels and the immunological alterations, the development of allergic sensitization is not a characteristic feature in STAT3-HIES patients, as it was detected earlier and also in our present study [39]. In our study the occurrence of allergic diseases (asthma, allergic rhinitis) differed significantly between the STAT3-HIES and AD groups. In STAT3-HIES group no relevant specific IgE positivity or clinically supported allergic sensitization were detected with serum specific $\operatorname{IgE}$ measurement as well as with skin prick test. Different mechanisms can stand in the background. In STAT3-HIES patients defective IL-10 signalling in dendritic cells has been detected, and since it is crucial for generating inducible regulative $\mathrm{T}$ cells (Treg), it can result in inappropriate Th2-Treg balance, in impaired $\mathrm{T}$ cell proliferation, in altered suppression of cytokine production, and in higher IgE levels and eosinophilia [40]. Another workgroup suggests that compromised mast cell granulation and basophil activation may play an important role in the prevention of allergic sensitization development [41]. We approached the question from another side, whether well-functioning physicochemical barriers can prevent sensitization in a disorder where immunological alterations would predispose to allergic diseases. We suggest that the normal physicochemical skin barrier functions, together with the above mentioned other mechanisms, play role in the prevention of these patients from the development of allergic sensitization, and this phenomenon can further strengthen the significant connection between skin barrier function and allergic sensitization.

\section{Conclusion}

In conclusion our study demonstrated for the first time that skin barrier functions of STAT3-HIES patients are not damaged, and are similar to those of healthy controls, as well as differ significantly from the altered skin barrier functions of AD patients. Since barrier defects are important in the pathogenesis of AD skin lesions and seem to be missing in STAT3HIES, this may indicate different pathogenesis and may explain the earlier detected clinical differences between the eczematoid skin lesions in these two disorders. These findings also suggest a distinct dermatological therapy for skin lesions of STAT3-HIES patients from the usual AD therapies, since barrier replacement therapies probably are not necessary. It also raises the question whether the 'AD-like skin lesions' nomenclature is correct in STAT3-HIES. Moreover our results provide a possible explanation why allergic sensitization is missing or not common in STAT3-HIES patients, in spite of their high serum IgE level and immunological alterations. Furthermore, our investigation supplies additional data to support those findings that suggest a strong connection between the skin barrier alterations and the development of allergic sensitization. A limitation of this study is the relatively low number of STAT3-HIES patients (since there are only 9 registered STAT3-HIES patients in Hungary), which indicates the need for further investigations, but the significant results can strengthen our main hypothesis.

Acknowledgments This work was supported by the Hungarian Research Grants (OTKA K108421, TÁMOP-4.2.2.A-11/1/KONV-20120023-“DEFENSE-NET”, OTKA K101761, LP003/2011).

Conflict of Interest The authors declare that they have no conflict of interest.

\section{References}

1. Minegishi Y. Hyper-IgE syndrome. Curr Opin Immunol. 2009;21(5):487-92.

2. Buckley RH. The hyper-IgE syndrome. Clin Rev Allergy Immunol. 2001;20(1):139-54.

3. Minegishi Y, Saito M, Tsuchiya S, Tsuge I, Takada H, Hara T, et al. Dominant-negative mutations in the DNA-binding domain of STAT3 cause hyper-IgE syndrome. Nature. 2007;448(7157): 1058-62.

4. Holland SM, DeLeo FR, Elloumi HZ, Hsu AP, Uzel G, Brodsky N, et al. STAT3 mutations in the hyper-IgE syndrome. N Engl J Med. 2007;357(16):1608-19.

5. Renner ED, Puck JM, Holland SM, Schmitt M, Weiss M, Frosch $\mathrm{M}$, et al. Autosomal recessive hyperimmunoglobulin E syndrome: a distinct disease entity. J Pediatr. 2004;144(1):93-9.

6. Minegishi Y, Saito M, Morio T, Watanabe K, Agematsu K, Tsuchiya S, et al. Human tyrosine kinase 2 deficiency reveals its requisite roles in multiple cytokine signals involved in innate and acquired immunity. Immunity. 2006;25(5):745-55.

7. Schimke LF, Sawalle-Belohradsky J, Roesler J, Wollenberg A, Rack A, et al. Diagnostic approach to the hyper-IgE syndromes: immunological and clinical key findings to differentiate hyper-IgE syndromes from atopic dermatitis. J Allergy Clin Immunol. 2010;126(3):611-7.

8. Milner JD, Brenchley JM, Laurence A, Freeman AF, Hill BJ, et al. Impaired $\mathrm{T}_{\mathrm{H}} 17$ cell differentiation in subjects with autosomal dominant hyper-IgE syndrome. Nature. 2008;452(7188):773-6.

9. de Beaucoudry L, Puel A, Filipe-Santos O, Cobat A, Ghandil P, et al. Mutations in STAT3 and IL12RB1 impair the development of human IL17-producing T cells. J Exp Med. 2008;205(7):1543-50. 
10. Minegishi Y, Saito M. Cutaneous manifestations of Hyper IgE syndrome. Allergol Int. 2012;61(2):191-6.

11. Maintz L, Novak N. Getting more and more complex: the pathophysiology of atopic eczema. Eur J Dermatol. 2007;17(4):267-83.

12. Boguniewicz M, Leung DY. Atopic dermatitis: a disease of altered skin barrier and immune dysregulation. Immunol Rev. 2011;242(1): 233-46.

13. Irvine $\mathrm{AD}, \mathrm{McLean} \mathrm{WH}$, Leung DY. Filaggrin mutations associated with skin and allergic diseases. N Engl J Med. 2011;365(14):131527.

14. Novak N, Leung DY. Advances in atopic dermatitis. Curr Opin Immunol. 2011;23(6):778-83.

15. Zold E, Szodoray P, Kappelmayer J, Gaal J, Csathy L, Barath S, et al. Impaired regulatory T-cell homeostasis due to vitamin D deficiency in undifferentiated connective tissue disease. Scand J Rheumatol. 2010;39(6):490-7.

16. Sano $\mathrm{Y}$, Masuda $\mathrm{K}$, Tamagawa-Mineoka $\mathrm{R}$, Matsunaka $\mathrm{H}$, Murakami Y, Yamashita R, et al. Thymic stromal lymphopoietin expression is increased in the horny layer of patients with atopic dermatitis. Clin Exp Immunol. 2013;171(3):330-7.

17. Eberting CL, Davis J, Puck JM, Holland SM, Turner ML. Dermatitis and the newborn rash of hyper-IgE syndrome. Arch Dermatol. 2004;140(9):1119-25.

18. Olaiwan A, Chandesris MO, Fraitag S, Lortholary O, Hermine O, Fischer A, et al. Cutaneous findings in sporadic and familial autosomal dominant hyper-IgE syndrome: a retrospective, single-center study of 21 patients diagnosed using molecular analysis. J Am Acad Dermatol. 2011;65(6):1167-72.

19. Erlewyn-Lajeunesse MD. Hyperimmunoglobulin-E syndrome with recurrent infection: a review of current opinion and treatment. Pediatr Allergy Immunol. 2000;11(3):133-41.

20. Bos JD. Atopiform dermatitis. Br J Dermatol. 2002;147(3):426-9.

21. Minegishi Y, Saito M, Nagasawa M, Takada H, Hara T, et al. Molecular explanation for the contradiction between systemic Th17 defect and localized bacterial infection in hyper-IgE syndrome. J Exp Med. 2009;206(6):1291-301.

22. Jakasa I, Koster ES, Calkoen F, McLean WH, Campbell LE, Bos $\mathrm{JD}$, et al. Skin barrier function in healthy subjects and patients with atopic dermatitis in relation to filaggrin loss-of-function mutations. J Invest Dermatol. 2011;131(2):540-2.

23. Nemoto-Hasebe I, Akiyama M, Nomura T, Sandilands A, McLean WH, Shimizu H. Clinical severity correlates with impaired barrier in filaggrin-related eczema. J Invest Dermatol. 2009;129(3):682-9.

24. Furuse M, Hata M, Furuse K, Yoshida Y, Haratake A, Sugitani Y, et al. Claudin-based tight junctions are crucial for the mammalian epidermal barrier: a lesson from claudin-1-deficient mice. J Cell Biol. 2002;156(6):1099-111.

25. Elias PM, Matsuyoshi N, Wu H, Lin C, Wang ZH, Brown BE, et al. Desmoglein isoform distribution affects stratum corneum structure and function. J Cell Biol. 2001;153(2):243-9.

26. Rippke F, Schreiner V, Schwanitz HJ. The acidic milieu of the horny layer: new findings on the physiology and pathophysiology of skin pH. Am J Clin Dermatol. 2002;3(4):261-72.
27. Yosipovitch G, Maibach HI. Significance of skin surface $\mathrm{pH}$. Harefuah. 1996;130(7):478-80.

28. Rippke F, Schreiner V, Doering T, Maibach HI. Stratum corneum $\mathrm{pH}$ in atopic dermatitis: impact on skin barrier function and colonization with Staphylococcus Aureus. Am J Clin Dermatol. 2004;5(4):217-23.

29. Smeekens SP, Huttenhower C, Riza A, van de Veerdonk FL, Zeeuwen PL, Schalkwijk J, et al. Skin Microbiome Imbalance in Patients with STAT1/STAT3 Defects Impairs Innate Host Defense Responses. J Innate Immun 2013.

30. Sandilands A, Sutherland C, Irvine AD, McLean WH. Filaggrin in the frontline: role in skin barrier function and disease. J Cell Sci. 2009; 122(Pt 9):1285-94.

31. McLoone P, Simics E, Barton A, Norval M, Gibbs NK. An action spectrum for the production of cis-urocanic acid in human skin in vivo. J Invest Dermatol. 2005;124(5):1071-4.

32. Demehri S, Liu Z, Lee J, Lin MH, Crosby SD, Roberts CJ, et al. Notch-deficient skin induces a lethal systemic Blymphoproliferative disorder by secreting TSLP, a sentinel for epidermal integrity. PLoS Biol. 2008;6(5), e123.

33. Lee EB, Kim KW, Hong JY, Jee HM, Sohn MH, Kim KE. Increased serum thymic stromal lymphopoietin in children with atopic dermatitis. Pediatr Allergy Immunol. 2010;21(2 Pt 2): e457-460.

34. Alysandratos KD, Angelidou A, Vasiadi M, Zhang B, Kalogeromitros D, Katsarou-Katsari A, et al. Increased affected skin gene expression and serum levels of thymic stromal lymphopoietin in atopic dermatitis. Ann Allergy Asthma Immunol. 2010;105(5):403-4.

35. Romagnani $\mathrm{S}$. The increased prevalence of allergy and the hygiene hypothesis: missing immune deviation, reduced immune suppression, or both? Immunology. 2004;112(3):352-63.

36. De Benedetto A, Kubo A, Beck LA. Skin barrier disruption: a requirement for allergen sensitization? J Invest Dermatol. 2012;132(3 Pt 2):949-63.

37. Weidinger S, Illig T, Baurecht $\mathrm{H}$, Irvine AD, Rodriguez E, DiazLacava A, et al. Loss-of-function variations within the filaggrin gene predispose for atopic dermatitis with allergic sensitizations. J Allergy Clin Immunol. 2006;118(1):214-9.

38. Novak N, Baurecht H, Schafer T, Rodriguez E, Wagenpfeil S, Klopp N, et al. Loss-of-function mutations in the filaggrin gene and allergic contact sensitization to nickel. J Invest Dermatol. 2008;128(6):1430-5.

39. Boos AC, Hagl B, Schlesinger A, Halm BE, Ballenberger N, Pinarci M, et al. Atopic dermatitis, STAT3- and DOCK8-hyper$\mathrm{IgE}$ syndromes differ in IgE-based sensitization pattern. Allergy. 2014;69(7):943-53.

40. Saito M, Nagasawa M, Takada H, Hara T, Tsuchiya S, Agematsu K, et al. Defective IL-10 signaling in hyper-IgE syndrome results in impaired generation of tolerogenic dendritic cells and induced regulatory T cells. J Exp Med. 2011;208(2):235-49.

41. Siegel AM, Stone KD, Cruse G, Lawrence MG, Olivera A, et al. Diminished allergic disease in patients with STAT3 mutations reveals a role for STAT3 signaling in mast cell degranulation. $\mathrm{J}$ Allergy Clin Immunol. 2013;132(6):1388-96. 